\title{
Chromatic completion number of corona of path and cycle graphs
}

\author{
Johan Kok ${ }^{1 *}$
}

\begin{abstract}
Following the introduction of the notion of chromatic completion of a graph, this paper presents results for the chromatic completion number for the corona operations, $P_{n} \circ P_{m}$ and $P_{n} \circ C_{m}, n \geq 1$ and $m \geq 1$. From the aforesaid a general result for the chromatic completion number of $P_{n} \circ K_{m}$ came to the fore. The paper serves as a basis for further research with regards to the chromatic completion number of corona, join and other graph products.
\end{abstract}

\section{Keywords}

Chromatic completion number, chromatic completion graph, chromatic completion edge.

\section{AMS Subject Classification}

05C15, 05C38, 05C75, 05C85.

${ }^{1}$ Independent Mathematics Researcher, South Africa \& Department of Mathematics, CHRIST (Deemed to be a University), Bangalore, India. *Corresponding author: jacotype@gmail.com; johan.kok@christuniversity.in.

Article History: Received 5 October 2019; Accepted 12 December 2019

(C)2020 MJM.

\section{Contents}

1 Introduction ..1

2 Chromatic Completion Number of $P_{n} \circ P_{m} \ldots \ldots \ldots .2$

2.1 Corona of paths, $P_{n} \circ P_{m}, n=3 t, t=1,2,3, \ldots$, and $m$ is even . . . . . . . . . . . . 3

2.2 Corona of paths, $P_{n^{\prime}} \circ P_{m}, n^{\prime}=3 t+1, t=1,2,3, \ldots$, and $m$ is even . . . . . . . . . . . . 3

2.3 Corona of paths, $P_{n^{\prime}} \circ P_{m}, n^{\prime}=3 t+2, t=1,2,3, \ldots$ and $m$ is even . . . . . . . . . . . . 3

2.4 Corona of paths, $P_{n} \circ P_{m}, n=3 t, t=1,2,3, \ldots$, and $m$ is odd . . . . . . . . . . . . . . . 4

3 Chromatic Completion Number of $P_{n} \circ C_{m} \ldots \ldots \ldots .4$

3.1 Corona $P_{n} \circ C_{m}, m \in O_{1}$ and $n=4 k, k=1,2,3, \ldots .4$

3.2 Corona $P_{n^{\prime}} \circ C_{m}, m \in O_{1}$ and $n^{\prime}=4 k+1$, $k=1,2,3, \ldots \ldots \ldots \ldots \ldots \ldots \ldots \ldots \ldots \ldots \ldots$

3.3 Corona $P_{n^{\prime}} \circ C_{m}, m \in O_{1}$ and $n^{\prime}=4 k+2$, $k=1,2,3, \ldots \ldots \ldots \ldots \ldots \ldots \ldots \ldots \ldots \ldots \ldots \ldots$

3.4 Corona $P_{n^{\prime}} \circ C_{m}, m \in O_{1}$ and $n^{\prime}=4 k+3$, $k=1,2,3, \ldots \ldots \ldots \ldots \ldots$

4

5

Conclusion.................................. 5

5 Acknowledgments $\ldots \ldots \ldots \ldots \ldots \ldots \ldots \ldots \ldots \ldots \ldots, 6$

References...................................6

\section{Introduction}

For general notation and concepts in graphs see $[1,2,5]$. It is assumed that the reader is familiar with the concept of graph coloring. Recall that in a proper coloring of $G$ all edges are good i.e. $u v \Leftrightarrow c(u) \neq c(v)$. For any proper coloring $\varphi(G)$ of a graph $G$ the addition of all good edges, if any, is called the chromatic completion of $G$ in respect of $\varphi(G)$. The additional edges are called chromatic completion edges. The set of such chromatic completion edges is denoted by, $E_{\varphi}(G)$. The resultant graph $G_{\varphi}$ is called a chromatic completion graph of $G$. See [3] for an introduction to chromatic completion of a graph.

The chromatic completion number of a graph $G$ denoted by, $\zeta(G)$ is the maximum number of good edges that can be added to $G$ over all chromatic colorings ( $\chi$-colorings). Hence, $\zeta(G)=\max \left\{\left|E_{\chi}(G)\right|:\right.$ over all $\left.\varphi_{\chi}(G)\right\}$.

A $\chi$-coloring which yields $\zeta(G)$ is called a Lucky $\chi$ coloring or simply, a Lucky coloring and is denoted by, $\varphi_{\mathscr{L}}(G)$. The resultant graph $G_{\zeta}$ is called a minimal chromatic completion graph of $G$. It is trivially true that $G \subseteq G_{\zeta}$. Furthermore, the graph induced by the set of completion edges, $\left\langle E_{\chi}\right\rangle$ is a subgraph of the complement graph, $\bar{G}$. See [4] for the notion of stability in respect of chromatic completion.

Recall that perfect Lucky $\chi$-coloring ${ }^{1}$ of a graph $G$ is a

\footnotetext{
${ }^{1}$ Note that for many graphs a perfect Lucky coloring is equivalent to an
} 
graph for which the vertex $V(G)$ can be partitioned in accordance to Lucky's theorem i.e. in the Lucky partition form,

$$
\begin{aligned}
& \{\underbrace{\left\{\left\lfloor\frac{n}{\chi(G)}\right\rfloor \text {-element }\right\},\left\{\left\lfloor\frac{n}{\chi(G)}\right\rfloor \text {-element }\right\}, \ldots,\left\{\left\lfloor\frac{n}{\chi(G)}\right\rfloor \text {-element }\right\}}_{(\chi(G)-r)-\text { subsets }}, \\
& \underbrace{\left\{\left\lceil\frac{n}{\chi(G)}\right\rceil \text {-element }\right\},\left\{\left\lceil\frac{n}{\chi(G)}\right\rceil \text {-element }\right\}, \ldots,\left\{\left\lceil\frac{n}{\chi(G)}\right\rceil \text {-element }\right\}}_{(r>0) \text {-subsets }}\} \text {. }
\end{aligned}
$$

Else, any graph is always near Lucky $\chi$-colorable (similar to near equitable colorable). The vertex partition which approximates a Lucky partition closest is called an optimal nearcompletion $\chi$-partition. See $[3,4]$. The number of times a color $c_{i}$ is assigned in a graph coloring is denoted by, $\theta_{G}\left(c_{i}\right)$. If the graph context is clear we abbreviate as, $\theta\left(c_{i}\right)$.

Various graph parameters have been studied in respect of sensitivity (how critical) the parameters are in respect of edge deletion, edge addition, vertex deletion or vertex insertion and alike. Note that after chromatic completion of a graph $G$ which has been assigned a chromatic coloring $(\chi(G)$ colors), the chromatic completion graph itself has chromatic number, $\chi(G)$. However, the addition of one or more further edges will result in an increase in chromatic number. If vertices and edges in a graph $G$ represent modules (or entities) and initial linkages in machine learning or artificial intelligence configurations, then:

(a) Different color classes could signal destructive linkages which may not occur and,

(b) Maximum permissible linkages may be needed to enhance machine learning or artificial intelligence interactive learning. (c) In such application the chromatic completion number of $G$ signals the critical threshold.

Similar applications can be visioned. This justifies further research into this parameter.

\section{Chromatic Completion Number of $P_{n} \circ P_{m}$}

Recall that the corona between graph $G$ or order $n$ and graph $H$ of order $m$ is obtained by taking $n$ copies of $H$ say, $H_{1}$, $H_{2}, H_{3}, \ldots, H_{n}$ and adding the edges, $v_{i} u_{i, j}, i=1,2,3, \ldots, n$ and $j=1,2,3, \ldots, m$. Put differently, $\forall v_{i}$ construct $v_{i}+H_{i}$ to $G$. We say, $H$ has been corona'd with $G$.

A path graph (or simply, a path) of order $n$ denoted by, $P_{n}$, is a graph on $n \geq 1$ vertices say, $V\left(P_{n}\right)=\left\{v_{1}, v_{2}, v_{3}, \ldots, v_{n}\right\}$ and $n-1$ edges namely, $E\left(P_{n}\right)=\left\{v_{i} v_{i+1}: i=1,2,3, \ldots, n-\right.$ $1\}$. In this section we denote, $V\left(P_{n}\right)=\left\{v_{1}, v_{2}, v_{3}, \ldots, v_{n}\right\}$ and $V\left(P_{m}\right)=\left\{u_{i, 1}, u_{i, 2}, u_{i, 3}, \ldots, u_{i, m}\right\}, i=1,2,3, \ldots, n$ and the edges accordingly. The corona operator (not necessarily commutative) will be, $P_{n} \circ P_{m}$.

equitable $\chi$-coloring. Since it is not generally the case the alias is meant to associate the paper with Lucky's Theorem and the notion of chromatic completion in $[3,4]$.
For $P_{1} \circ P_{1}$ it follows that $\zeta\left(P_{1} \circ P_{1}\right)=0$ because, $P_{1} \circ P_{1} \cong$ $K_{2}$. Similarly, $\zeta\left(P_{1} \circ P_{2}\right)=0$ because, $P_{1} \circ P_{2} \cong K_{3}$. Also, $\zeta\left(P_{1} \circ P_{3}\right)=0$ because, $c\left(u_{1,1}\right)=c\left(u_{1,3}\right)$ in any perfect Lucky $\chi$-coloring. We recall two important results from [3].

Lemma 2.1. [3] For a chromatic coloring $\varphi: V(G) \mapsto \mathscr{C}$ a pseudo completion graph, $H(\varphi)=K_{n_{1}, n_{2}, n_{3}, \ldots, n_{\chi}}$ exists such that,

$\varepsilon(H(\varphi))-\varepsilon(G)=\sum_{i=1}^{\chi-1} \theta_{G}\left(c_{i}\right) \theta_{G}\left(c_{j}\right)_{(j=i+1, i+2, i+3, \ldots, \chi)}-\varepsilon(G)$

$\leq \zeta(G)$.

Corollary 2.1. [3] Let $G$ be a graph. Then

$\zeta(G)=\max \{\varepsilon(H(\varphi))-\varepsilon(G):$ over all $\varphi: V(G) \mapsto \mathscr{C}\}$.

Corollary 2.2. (a) For $P_{1} \circ P_{m}, m \geq 4$ it follows that, $\zeta\left(P_{1} \circ\right.$ $\left.P_{m}\right)=\left\lceil\frac{m}{2}\right\rceil\left\lfloor\frac{m}{2}\right\rfloor-(m-1)$.

(b) For $P_{n} \circ P_{1}, n \geq 2$ it follows that, $\zeta\left(P_{n} \circ P_{1}\right)=n^{2}-n+1$.

Proof. (a) Since $\chi\left(P_{1} \circ P_{m}\right)=3$ let $c\left(v_{1}\right)=c_{1}$. Clearly $P_{m}$ can be assigned a perfect Lucky 2-coloring with the set of colors, $\left\{c_{2}, c_{3}\right\}$. Because $P_{1} \circ P_{m} \cong P_{1}+P_{m}$, no chromatic completion edges, $v_{1} u_{1, i}, 1 \leq i \leq m$ can be added. Hence, $\zeta\left(P_{1} \circ P_{m}\right)=\zeta\left(P_{m}\right)=\left\lceil\frac{m}{2}\right\rceil\left\lfloor\frac{m}{2}\right\rfloor-(m-1)$. See [3].

(b) Because $P_{n} \circ P_{1}$ is a tree on $2 n$ vertices the result is immediate from Lemma 2.1 and Corollary 2.1.

Note the subtlety in the proof above i.e. $P_{1} \circ P_{m}$ is not perfect Lucky 3-colorable. However, the induced subgraph $P_{m}$ of $P_{1} \circ P_{m}$, is perfect 2-colorable. Such vertex partition is called an optimal near-completion $\chi$-partition.

Proposition 2.1. For $P_{2} \circ P_{m}, m \geq 2$ it follows that,

$$
\zeta\left(P_{2} \circ P_{m}\right)= \begin{cases}\frac{5 m^{2}}{4}-m+2, & \text { if } m \text { is even, } \\ \frac{5 m^{2}+2 m+1}{4}-m, & \text { if } m \text { is odd. }\end{cases}
$$

Proof. Part 1. Consider $m$ is even. Without loss of generality, let $c\left(v_{1}\right)=c_{1}, c\left(v_{2}\right)=c_{2}$. Also without loss of generality let $c\left(u_{1, j}\right)=c_{2}, j=1,3,5, \ldots,(m-1)$ and $c\left(u_{1, j}\right)=c_{3}, j=$ $2,4,6, \ldots, m$. Also let, $c\left(u_{2, j}\right)=c_{1}, j=1,3,5, \ldots,(m-1)$ and $c\left(u_{2, j}\right)=c_{3}, j=2,4,6, \ldots, m$. Therefore, $\theta\left(c_{1}\right)=\frac{m}{2}+1$, $\theta\left(c_{2}\right)=\frac{m}{2}+1$ and $\theta\left(c_{3}\right)=m$. Clearly for $m \geq 6$, the vertex partition is an optimal near-completion $\chi$-partition.

From Lemma 2.1 and Corollary 2.1 it follow that,

$\zeta\left(P_{2} \circ P_{m}\right)=\left(\frac{m}{2}+1\right)^{2}+2 m\left(\frac{m}{2}+1\right)-(4 m-1)=\frac{5 m^{2}}{4}-m+2$.

Part 2. Consider $m$ is odd. Without loss of generality, let $c\left(v_{1}\right)=c_{1}, c\left(v_{2}\right)=c_{2}$. Also without loss of generality let $c\left(u_{1, j}\right)=c_{2}, j=1,3,5, \ldots, m$ and $c\left(u_{1, j}\right)=c_{3}, j=2,4,6, \ldots$, $(m-1)$. Also let, $c\left(u_{2, j}\right)=c_{1}, j=1,3,5, \ldots, m$ and $c\left(u_{2, j}\right)=$ $c_{3}, j=2,4,6, \ldots,(m-1)$. Therefore, $\theta\left(c_{1}\right)=\frac{m+1}{2}+1, \theta\left(c_{2}\right)=$ $\frac{m+1}{2}+1$ and $\theta\left(c_{3}\right)=m-1$. Clearly for $m \geq 7$, the vertex partition is an optimal near-completion $\chi$-partition.

From Lemma 2.1 and Corollary 2.1 it follow that, 
$\zeta\left(P_{2} \circ P_{m}\right)=\left(\frac{m+1}{2}+1\right)^{2}+2(m-1)\left(\frac{m+1}{2}+1\right)-(4 m-1)=$ $\frac{5 m^{2}+2 m+1}{4}-m$.

2.1 Corona of paths, $P_{n} \circ P_{m}, n=3 t, t=1,2,3, \ldots$, and $m$ is even

The families of paths considered first, i.e. $n=0(\bmod 3)$ is meant to provide the foundation for $n=1(\bmod 3)$ or $n=$ $2(\bmod 3)$ as well as for the corona, $P_{n} \circ C_{m}, m$ is even.

Proposition 2.2. For $P_{n} \circ P_{m}, n=3 t, t=1,2,3, \ldots$, and $m$ is even it follows that, $\zeta\left(P_{n} \circ P_{m}\right)=\frac{n^{2}(m-1)^{2}}{3}-2 n m+1$.

Proof. Since $\chi\left(P_{n} \circ P_{m}\right)=3$, color the vertices $v_{i}, i=1,2,3, \ldots$, $n$ as follows, $c\left(v_{i+3 j}\right)=c_{i}, i=1,2,3$ and $j=0,1,2, \ldots,(t-$ $1)$. Furthermore, color the vertices of the $n$ copies of $P_{m}$, as follows. For $j=0,1,2, \ldots,(t-1)$ and $k_{1}=1,3,5, \ldots,(m-1)$, $k_{2}=2,4,6, \ldots, m$, let:

$$
\begin{aligned}
& c\left(u_{1+3 j, k_{1}}\right)=c_{2}, c\left(u_{1+3 j, k_{2}}\right)=c_{3}, \\
& c\left(u_{2+3 j, k_{1}}\right)=c_{1}, c\left(u_{2+3 j, k_{2}}\right)=c_{3}, \\
& c\left(u_{3+3 j, k_{1}}\right)=c_{1}, c\left(u_{3+3 j, k_{2}}\right)=c_{2} .
\end{aligned}
$$

It follows easily that, $\theta\left(c_{1}\right)=\theta\left(c_{2}\right)=\theta\left(c_{3}\right)$ which is a perfect Lucky 3-coloring of $P_{n} \circ P_{m}$.

Furthermore, $\theta\left(c_{i}\right)=2 t \frac{m}{2}+t=\frac{n(m-1)}{3}, i=1,2,3$. Also, $\varepsilon\left(P_{n} \circ P_{m}\right)=(n-1)+n m+n(m-1)=2 n m-1$. Therefore, from Lemma 2.1 and Corollary 2.1 it follow that, $\zeta\left(P_{n} \circ P_{m}\right)=$ $\frac{n^{2}(m-1)^{2}}{3}-2 n m+1$.

2.2 Corona of paths, $P_{n^{\prime}} \circ P_{m}, n^{\prime}=3 t+1, t=1,2,3, \ldots$, and $m$ is even

Through immediate induction it follows we just need to extend path $P_{n}$ in Proposition 2.2 to path, $P_{n+1}$ and derive the result through similar reasoning. The result is presented as a corollary of Proposition 2.2.

Corollary 2.3. For $P_{n^{\prime}} \circ P_{m}, n^{\prime}=3 t+1, t=1,2,3, \ldots$, and $m$ is even it follows that, $\zeta\left(P_{n^{\prime}} \circ P_{m}\right)=2\left(\frac{n(m-1)}{3}+1\right)\left(\frac{n(m-1)}{3}+\right.$ $\left.\frac{m}{2}\right)+\left(\frac{n(m-1)}{3}+\frac{m}{2}\right)^{2}-2 m(n+1)+1$.

Proof. Following the coloring protocol in Proposition 2.2 and without loss of generality let, $c\left(v_{n+1}\right)=c_{1}$. It implies that, $c\left(u_{(n+1), i}\right)=c_{2}, i=1,3,5, \ldots,(m-1)$ and $c\left(u_{(n+1), i}\right)=$ $c_{3}, i=2,4,6, \ldots, m$. Hence, $\theta\left(c_{1}\right)=\frac{n(m-1)}{3}+1, \theta\left(c_{2}\right)=$ $\frac{n(m-1)}{3}+\frac{m}{2}$ and $\theta\left(c_{3}\right)=\frac{n(m-1)}{3}+\frac{m}{2}$. Clearly, for sufficiently large $m$ the coloring is not a perfect Lucky 3-coloring. However, the vertex partition is an optimal near-completion $\chi$ partition. Therefore, the chromatic completion will yield the chromatic completion number.

Also, $\varepsilon\left(P_{n+1} \circ P_{m}\right)=2 n m-1+(1+m+(m-1))=2 m(n+$ 1) -1 . Finally,

$$
\begin{gathered}
\zeta\left(P_{n^{\prime}} \circ P_{m}\right)=\zeta\left(P_{n+1} \circ P_{m}\right)= \\
2\left(\frac{n(m-1)}{3}+1\right)\left(\frac{n(m-1)}{3}+\frac{m}{2}\right)+\left(\frac{n(m-1)}{3}+\frac{m}{2}\right)^{2}-2 m(n+1)+1 .
\end{gathered}
$$

2.3 Corona of paths, $P_{n^{\prime}} \circ P_{m}, n^{\prime}=3 t+2, t=1,2,3, \ldots$ and $m$ is even

Through immediate induction it follows we just need to extend path $P_{n+1}$ in Corollary 2.3 to path, $P_{n+2}$ and derive the result through similar reasoning. The result is presented as a corollary of Proposition 2.2.

Corollary 2.4. For $P_{n^{\prime}} \circ P_{m}, n^{\prime}=3 t+2, t=1,2,3, \ldots$, and $m$ is even it follows that, $\zeta\left(P_{n^{\prime}} \circ P_{m}\right)=\left(\frac{n(m-1)}{3}+\frac{m}{2}+1\right)\left(\frac{n(m-1)}{3}+\right.$ $\left.\frac{m}{2}+\frac{m}{2}\right)+\left(\frac{n(m-1)}{3}+\frac{m}{2}+1\right)\left(\frac{n(m-1)}{3}+\frac{m}{2}+m\right)+\left(\frac{n(m-1)}{3}+\frac{m}{2}+\right.$ $\left.\frac{m}{2}\right)\left(\frac{n(m-1)}{3}+\frac{m}{2}+m\right)$

$-2 n m-4 m+1$.

Proof. Following the coloring protocol in Corollary 2.3 (extended from Proposition 2.2) and without loss of generality let, $c\left(v_{n+2}\right)=c_{2}$. It implies that, $c\left(u_{(n+2), i}\right)=c_{1}, i=$ $1,3,5, \ldots,(m-1)$ and $c\left(u_{(n+2), i}\right)=c_{3}, i=2,4,6, \ldots, m$. Hence, $\theta\left(c_{1}\right)=\frac{n(m-1)}{3}+\frac{m}{2}+1, \theta\left(c_{2}\right)=\frac{n(m-1)}{3}+\frac{m}{2}+\frac{m}{2}$ and $\theta\left(c_{3}\right)=$ $\frac{n(m-1)}{3}+\frac{m}{2}+m$. Clearly, for sufficiently large $m$ the coloring is not a perfect Lucky 3-coloring. However, the vertex partition is an optimal near-completion $\chi$-partition. Therefore, the chromatic completion will yield the chromatic completion number.

Also, $\varepsilon\left(P_{n+2} \circ P_{m}\right)=2 n m-1+2(1+m+(m-1))=2 n m+$ $4 m-1$. Finally,

$$
\begin{gathered}
\zeta\left(P_{n^{\prime}} \circ P_{m}\right)=\zeta\left(P_{n+2} \circ P_{m}\right)= \\
\left(\frac{n(m-1)}{3}+\frac{m}{2}+1\right)\left(\frac{n(m-1)}{3}+\frac{m}{2}+\frac{m}{2}\right)+\left(\frac{n(m-1)}{3}+\frac{m}{2}+\right. \\
1)\left(\frac{n(m-1)}{3}+\frac{m}{2}+m\right)+ \\
\left(\frac{n(m-1)}{3}+\frac{m}{2}+\frac{m}{2}\right)\left(\frac{n(m-1)}{3}+\frac{m}{2}+m\right)-\varepsilon\left(P_{n+2} \circ P_{m}\right)= \\
\left(\frac{n(m-1)}{3}+\frac{m}{2}+1\right)\left(\frac{n(m-1)}{3}+\frac{m}{2}+\frac{m}{2}\right)+\left(\frac{n(m-1)}{3}+\frac{m}{2}+\right. \\
1)\left(\frac{n(m-1)}{3}+\frac{m}{2}+m\right)+ \\
\left(\frac{n(m-1)}{3}+\frac{m}{2}+\frac{m}{2}\right)\left(\frac{n(m-1)}{3}+\frac{m}{2}+m\right)-2 n m-4 m+1 .
\end{gathered}
$$

The results can be summarized as a main result for $n=$ $i(\bmod 3)$.

Theorem 2.1. For $P_{n} \circ P_{m}, n \geq 3$ and $m$ is even it follows that,

(a) If $m=0(\bmod 3)$ then, $\zeta\left(P_{n} \circ P_{m}\right)=\frac{n^{2}(m-1)^{2}}{3}-2 n m+1$.

(b) If $m=1(\bmod 3)$ then,

$$
\begin{gathered}
\zeta\left(P_{n} \circ P_{m}\right)=2\left(\frac{(n-1)(m-1)}{3}+1\right)\left(\frac{9 n-1)(m-1)}{3}+\frac{m}{2}\right)+ \\
\left(\frac{(n-1)(m-1)}{3}+\frac{m}{2}\right)^{2}-2 m n+1 .
\end{gathered}
$$

(c) If $m=2(\bmod 3)$ then,

$$
\begin{gathered}
\zeta\left(P_{n} \circ P_{m}\right)=\left(\frac{(n-2)(m-1)}{3}+\frac{m}{2}+1\right)\left(\frac{(n-2)(m-1)}{3}+\frac{m}{2}+\frac{m}{2}\right)+ \\
\left(\frac{(n-2)(m-1)}{3}+\frac{m}{2}+1\right)\left(\frac{(n-2)(m-1)}{3}+\frac{m}{2}+m\right)+ \\
\left(\frac{(n-2)(m-1)}{3}+\frac{m}{2}+\frac{m}{2}\right)\left(\frac{(n-2)(m-1)}{3}+\frac{m}{2}+m\right)- \\
2 m(n-2)-4 m+1 .
\end{gathered}
$$


2.4 Corona of paths, $P_{n} \circ P_{m}, n=3 t, t=1,2,3, \ldots$, and $m$ is odd

The families of paths considered first, i.e. $n=0(\bmod 3)$ is meant to provide the foundation for $n=1(\bmod 3)$ or $n=$ $2(\bmod 3)$.

Proposition 2.3. For $P_{n} \circ P_{m}, n=3 t, t=1,2,3, \ldots$, and $m \geq$ $3, m$ is odd it follows that, $\zeta\left(P_{n} \circ P_{m}\right)=n^{2}(m+1)^{2}-2 n m+1$.

Proof. Since $\chi\left(P_{n} \circ P_{m}\right)=3$, color the vertices $v_{i}, i=1,2,3, \ldots$, $n$ as follows, $c\left(v_{i+3 j}\right)=c_{i}, i=1,2,3$ and $j=0,1,2, \ldots,(t-$ 1). Furthermore, color the vertices of the $n$ copies of $P_{m}$, as follows. For $j=0,1,2, \ldots,(t-1)$ and $k_{1}=1,3,5, \ldots, m$, $k_{2}=2,4,6, \ldots,(m-1)$, let:

$$
\begin{aligned}
& c\left(u_{1+3 j, k_{1}}\right)=c_{2}, c\left(u_{1+3 j, k_{2}}\right)=c_{3}, \\
& c\left(u_{2+3 j, k_{1}}\right)=c_{3}, c\left(u_{2+3 j, k_{2}}\right)=c_{1}, \\
& c\left(u_{3+3 j, k_{1}}\right)=c_{1}, c\left(u_{3+3 j, k_{2}}\right)=c_{2} .
\end{aligned}
$$

It follows easily that, $\theta\left(c_{1}\right)=\theta\left(c_{2}\right)=\theta\left(c_{3}\right)$ which is a perfect Lucky 3-coloring of $P_{n} \circ P_{m}$.

Furthermore, $\theta\left(c_{i}\right)=t\left(\left\lceil\frac{m}{2}\right\rceil+\left\lfloor\frac{m}{2}\right\rfloor\right)+t=\frac{n(m+1)}{3}, i=1,2,3$. Also, $\varepsilon\left(P_{n} \circ P_{m}\right)=(n-1)+n m+n(m-1)=2 n m-1$. Therefore, from Lemma 2.1 and Corollary 2.1 it follow that, $\zeta\left(P_{n} \circ\right.$ $\left.P_{m}\right)=n^{2}(m+1)^{2}-2 n m+1$.

For $P_{n^{\prime}} \circ P_{m}, n^{\prime}=3 t+1$ or $n^{\prime}=3 t+2, t=1,2,3, \ldots$, and $m \geq 3, m$ is odd, a corollary follows since the methodology of proof is similar to that in Subsections 2.2 and 2.3.

Corollary 2.5. For $P_{n^{\prime}} \circ P_{m}, t=1,2,3, \ldots$, and $m \geq 3, m$ is odd we have that:

(a) If $n^{\prime}=3 t+1$ then,

$$
\begin{gathered}
\zeta\left(P_{n^{\prime}} \circ P_{m}\right)=2\left(\frac{n(m+1)}{3}+1\right)\left(\frac{n(m+1)}{3}+\frac{m-1}{2}\right)+ \\
\left(\frac{n(m+1)}{3}+\frac{m+1}{2}\right)\left(\frac{n(m+1)}{3}+\frac{m-1}{2}\right)-2 m(n+1)+1 .
\end{gathered}
$$

(b) If $n^{\prime}=3 t+2$ then,

$$
\begin{gathered}
\zeta\left(P_{n^{\prime}} \circ P_{m}\right)=\left(\frac{n(m+1)}{3}+\frac{m-1}{2}+1\right)\left(\frac{n(m+1)}{3}+\frac{m+1}{2}+1\right)+ \\
\left(\frac{n(m+1)}{3}+\frac{m-1}{2}+1\right)\left(\frac{n(m+1)}{3}+m\right)+\left(\frac{n(m+1)}{3}+\frac{m+1}{2}+\right. \\
1)\left(\frac{n(m+1)}{3}+m\right)- \\
2 m(n+2)+1 .
\end{gathered}
$$

\section{Chromatic Completion Number of $P_{n} \circ C_{m}$}

A cycle graph (or simply, a cycle) of order $n$ denoted by, $C_{n}$, is a graph on $n \geq 1$ vertices say, $V\left(C_{n}\right)=\left\{v_{1}, v_{2}, v_{3}, \ldots, v_{n}\right\}$ and $n$ edges namely, $E\left(C_{n}\right)=\left\{v_{i} v_{i+1}: i=1,2,3, \ldots, n-1\right\} \cup$ $\left\{v_{n} v_{1}\right\}$.

In this section we denote, $V\left(C_{m}\right)=\left\{u_{i, 1}, u_{i, 2}, u_{i, 3}, \ldots, u_{i, m}\right\}$, $i=1,2,3, \ldots, n$ and the edges accordingly.

Theorem 3.1. For $P_{n} \circ C_{m}, n \geq 3$ and $m \geq 4$ is even it follows that, $\zeta\left(P_{n} \circ C_{m}\right)=\zeta\left(P_{n} \circ P_{m}\right)-n$.
Proof. Since $m$ is even, $c\left(u_{i, 1}\right) \neq c\left(u_{i, m}\right), \forall i$ in any proper coloring. Since edge $u_{i, 1} u_{i, m} \in E\left(C_{m}\right)$ it cannot be a chromatic completion edge as yielded in the chromatic completion of $P_{n} \circ P_{m}$. Therefore, the result is immediate.

The next general result provides for the result, $\zeta\left(P_{n} \circ\right.$ $\left.C_{3}\right)=(n-1)(6 n-1)$.

Theorem 3.2. For $P_{n} \circ K_{m}, n \geq 1, m \geq 1$, it follows that, $\zeta\left(P_{n} \circ K_{m}\right)=(n-1)\left(\frac{n m(m+1)}{2}-1\right)$.

Proof. Consider $P_{n} \circ K_{m}, n \geq 1, m \geq 1$. Since, $P_{n} \circ K_{m} \cong H$ where $H$ is the graph obtained from $n$ copies of $\left\langle\left\{v_{i}\right\} \cup\right.$ $\left.\left\{u_{i, j}\right\}_{\in V\left(K_{m, i}\right)}\right) \cong K_{(m+1), i}, i=1,2,3, \ldots, n, j=1,2,3, \ldots, m$ linked as a string by the edges $v_{i} v_{i+1}, i=1,2,3, \ldots,(n-$ $1)$, it follows that $\chi\left(P_{n} \circ K_{m}\right)=m+1$. Hence, $\theta\left(c_{i}\right)=n$, $1 \leq i \leq(m+1)$ which is a perfect Lucky $(m+1)$-coloring. Also, $\varepsilon\left(P_{n} \circ K_{m}\right)=\frac{n m(m+1)}{2}+(n-1)$. Thus, from Lemma 2.1 and Corollary 2.1 it follow that, $\zeta\left(P_{n} \circ K_{m}\right)=\left(\frac{n^{2} m(m+1)}{2}\right)-$ $\left(\frac{n m(m+1)}{2}+(n-1)\right)=(n-1)\left(\frac{n m(m+1)}{2}-1\right)$. Immediate induction ensures that the result holds for, $n, m \in \mathbb{N}$.

It follows easily that for, $P_{1} \circ G, G$ any graph, $\zeta\left(P_{1} \circ G\right)=$ $\zeta(G)$. The set of odd integers, $\{m \in \mathbb{N}: m \geq 3$ and $m$ is odd $\}$ will be partitioned into three sets. The sets are, $O_{1}=$ $\{3+6 t: t=0,1,2,3, \ldots\}, O_{2}=\{5+6 t: t=0,1,2, \ldots\}$ and $O_{3}=\{7+6 t: t=0,1,2, \ldots\}$.

3.1 Corona $P_{n} \circ C_{m}, m \in O_{1}$ and $n=4 k, k=1,2,3, \ldots$

First the cases $n=2,3$ will be presented.

Proposition 3.1. For $P_{2} \circ C_{m}, m \in O_{1}$ and $m \geq 9$ it follows that, $\zeta\left(P_{2} \circ C_{m}\right)=\frac{m(13 m-6)}{9}$.

Proof. Without loss of generality let $c\left(v_{1}\right)=c_{1}, c\left(v_{2}\right)=c_{2}$. Because $m \in O_{1}$ we have, $\theta\left(c_{1}\right)=\frac{m}{3}+1, \theta\left(c_{1}\right)=\frac{m}{3}+1$, $\theta\left(c_{3}\right)=\frac{2 m}{3}$ and $\theta\left(c_{4}\right)=\frac{2 m}{3}$ which is not a perfect Lucky 3coloring. However, the vertex partition is an optimal nearcompletion $\chi$-partition. Therefore, the chromatic completion will yield the chromatic completion number.

Also, $\varepsilon\left(P_{2} \circ C_{m}\right)=4 m+1$. From Lemma 2.1 and Corollary 2.1 it follow that, $\zeta\left(P_{2} \circ C_{m}\right)=\frac{13 m^{2}}{9}+\frac{10 m}{3}+1-(4 m+1)=$ $\frac{m(13 m-6)}{9}$.

Proposition 3.2. For $P_{3} \circ C_{m}, m \in O_{1}$ and $m \geq 9$ it follows that, $\zeta\left(P_{3} \circ C_{m}\right)=3\left(\left(\frac{2 m}{3}+1\right)^{2}+m\left(\frac{2 m}{3}+1\right)\right)-2(3 m+1)$.

Proof. Without loss of generality let $c\left(v_{1}\right)=c_{1}, c\left(v_{2}\right)=$ $c_{2}, c\left(v_{3}\right)=c_{3}$. Because $m \in O_{1}$ we have, $\theta\left(c_{1}\right)=\frac{2 m}{3}+1$, $\theta\left(c_{2}\right)=\frac{2 m}{3}+1, \theta\left(c_{3}\right)=\frac{2 m}{3}+1$ and $\theta\left(c_{4}\right)=\frac{3 m}{3}=m$ which is not a perfect Lucky 3-coloring. However, the vertex partition is an optimal near-completion $\chi$-partition. Therefore, the chromatic completion will yield the chromatic completion number.

Also, $\varepsilon\left(P_{3} \circ C_{m}\right)=2(3 m+1)$. From Lemma 2.1 and Corollary 2.1 it follow that, $\zeta\left(P_{3} \circ C_{m}\right)=3\left(\left(\frac{2 m}{3}+1\right)^{2}+m\left(\frac{2 m}{3}+\right.\right.$ $1))-2(3 m+1)$. 
Proposition 3.3. For $P_{n} \circ C_{m}, m \in O_{1}$ and $n=4 k, k=1,2,3, \ldots$ it follows that,

$\zeta\left(P_{n} \circ C_{m}\right)=\frac{3 n^{2}}{8}(m+1)^{2}-n(m+1)-(m-1)$.

Proof. Since $\chi\left(P_{n} \circ C_{m}\right)=4$, color the vertices $v_{i}$ as follows, $c\left(v_{i+4 j}\right)=c_{i}, i=1,2,3,4$ and $j=0,1,2, \ldots,(k-1)$. Furthermore, color the vertices of the $n$ copies of $C_{m}$, as follows. For $j=0,1,2, \ldots,(k-1)$ and $s_{1}=1,4,7, \ldots,(m-2)$, $s_{2}=2,5,8, \ldots,(m-1), s_{3}=3,6,9, \ldots, m$ let:

$$
\begin{aligned}
& c\left(u_{1+4 j, s_{1}}\right)=c_{2}, c\left(u_{1+4 j, s_{2}}\right)=c_{3}, c\left(u_{1+4 j, s_{3}}\right)=c_{4}, \\
& c\left(u_{2+4 j, s_{1}}\right)=c_{1}, c\left(u_{2+4 j, s_{2}}\right)=c_{3}, c\left(u_{2+4 j, s_{3}}\right)=c_{4}, \\
& c\left(u_{3+4 j, s_{1}}\right)=c_{1}, c\left(u_{3+4 j, s_{2}}\right)=c_{2}, c\left(u_{3+4 j, s_{3}}\right)=c_{4}, \\
& c\left(u_{4+4 j, s_{1}}\right)=c_{1}, c\left(u_{4+4 j, s_{2}}\right)=c_{2}, c\left(u_{4+4 j, s_{3}}\right)=c_{3} .
\end{aligned}
$$

Thus, the vertex partition is a perfect Lucky 4-partition yielding a perfect Lucky 4-coloring. Also, $\theta\left(c_{i}\right)=k(m+1), 1 \leq$ $i \leq 4$ and $\varepsilon\left(P_{n} \circ C_{m}\right)=n(m+1)+(m-1)$. From Lemma 2.1 and Corollary 2.1 it follow that, $\zeta\left(P_{n} \circ C_{m}\right)=6 k^{2}(m+1)^{2}-$ $n(m+1)-(m-1)=\frac{3 n^{2}}{8}(m+1)^{2}-n(m+1)-(m-1)$.

3.2 Corona $P_{n^{\prime}} \circ C_{m}, m \in O_{1}$ and $n^{\prime}=4 k+1$, $k=1,2,3, \ldots$.

Through immediate induction it follows that we just need to extend path $P_{n}$ in Proposition 3.3 to path $P_{n+1}$ and derive the result through similar reasoning.

Corollary 3.1. For $P_{n^{\prime}} \circ C_{m}, m \in O_{1}$ and $n^{\prime}=4 k+1, k=$ $1,2,3, \ldots$ it follows that,

$\zeta\left(P_{n^{\prime}} \circ C_{m}\right)=3\left[\left(\frac{n}{4}(m+1)+1\right)\left(\frac{n}{4}(m+1)+\frac{m}{3}\right)+\left(\frac{n}{4}(m+1)+\right.\right.$ $\left.\left.\frac{m}{3}\right)^{2}\right]-n(m+1)-3 m$.

Proof. Following the coloring protocol in Proposition 3.3 and without loss of generality let, $c\left(v_{n+1}\right)=c_{1}$. It implies that $c\left(u_{n+1, i}\right)=c_{2}, i=1,4,7, \ldots,(m-2), c\left(u_{n+1, i}\right)=c_{3}, i=$ $2,5,8, \ldots,(m-1), c\left(u_{n+1, i}\right)=c_{4}, i=3,6,8, \ldots, m$. Hence, $\theta\left(c_{1}\right)=k(m+1)+1, \theta\left(c_{2}\right)=\theta\left(c_{3}\right)=\theta\left(c_{4}\right)=k(m+1)+\frac{m}{3}$ which is not a perfect Lucky 4-coloring. However, the vertex partition is an optimal near-completion $\chi$-partition. Therefore, the chromatic completion will yield the chromatic completion number.

Also, $\varepsilon\left(P_{n^{\prime}} \circ P_{m}\right)=n(m+1)+(m-1)+(m+1)+m=n(m+$ $1)+3 m$. From Lemma 2.1 and Corollary 2.1 it follow that, $\zeta\left(P_{n^{\prime}} \circ C_{m}\right)=3\left[\left(\frac{n}{4}(m+1)+1\right)\left(\frac{n}{4}(m+1)+\frac{m}{3}\right)+\left(\frac{n}{4}(m+1)+\right.\right.$ $\left.\left.\frac{m}{3}\right)^{2}\right]-n(m+1)-3 m$.

3.3 Corona $P_{n^{\prime}} \circ C_{m}, m \in O_{1}$ and $n^{\prime}=4 k+2$, $k=1,2,3, \ldots$

Through immediate induction it follows that we just need to extend path $P_{n}$ in Proposition 3.3 to path $P_{n+2}$ and derive the result through similar reasoning.

Corollary 3.2. For $P_{n^{\prime}} \circ C_{m}, m \in O_{1}$ and $n^{\prime}=4 k+2, k=$ $1,2,3, \ldots$ it follows that,

$\zeta\left(P_{n^{\prime}} \circ C_{m}\right)=\left(\left(\frac{n}{4}(m+1)+\frac{m}{3}+1\right)\left(\frac{5 n}{4}(m+1)+3 m+1\right)+\right.$ $\left(\frac{n}{4}(m+1)+\frac{2 m}{3}\right)^{2}-n(m+1)-5 m-1$.
Proof. Consider the coloring of $P_{n+1}$ in Corollary 3.2. Follow the coloring protocol in Proposition 3.3 and without loss of generality let, $c\left(v_{n+2}\right)=c_{2}$. It implies that $c\left(u_{n+2, i}\right)=c_{1}$, $i=1,4,7, \ldots,(m-2), c\left(u_{n+1, i}\right)=c_{3}, i=2,5,8, \ldots,(m-1)$, $c\left(u_{n+1, i}\right)=c_{4}, i=3,6,8, \ldots, m$. Hence, $\theta\left(c_{1}\right)=k(m+1)+$ $\frac{m}{3}+1, \theta\left(c_{2}\right)=k(m+1)+\frac{m}{3}+1, \theta\left(c_{3}\right)=\theta\left(c_{4}\right)=k(m+$ $1)+\frac{2 m}{3}$ which is not a perfect Lucky 4-coloring. However, the vertex partition is an optimal near-completion $\chi$-partition. Therefore, the chromatic completion will yield the chromatic completion number.

Also, $\varepsilon\left(P_{n^{\prime}} \circ P_{m}\right)=n(m+1)+5 m+1$. From Lemma 2.1 and Corollary 2.1 it follow that, $\zeta\left(P_{n^{\prime}} \circ C_{m}\right)=\left(\left(\frac{n}{4}(m+1)+\right.\right.$ $\left.\frac{m}{3}+1\right)\left(\frac{5 n}{4}(m+1)+3 m+1\right)+\left(\frac{n}{4}(m+1)+\frac{2 m}{3}\right)^{2}-n(m+1)-$ $5 m-1$.

3.4 Corona $P_{n^{\prime}} \circ C_{m}, m \in O_{1}$ and $n^{\prime}=4 k+3$, $k=1,2,3, \ldots$.

Through immediate induction it follows that we just need to extend path $P_{n}$ in Proposition 3.3 to path $P_{n+3}$ and derive the result through similar reasoning.

Corollary 3.3. For $P_{n^{\prime}} \circ C_{m}, m \in O_{1}$ and $n^{\prime}=4 k+3, k=$ $1,2,3, \ldots$ it follows that,

$\zeta\left(P_{n^{\prime}} \circ C_{m}\right)=3\left[\left(k(m+1)+\frac{2 m}{3}+1\right)^{2}+\left(k(m+1)+\frac{2 m}{3}+1\right)(k(m+\right.$ 1) $+m)]-n(m+1)-7 m-2$.

Proof. Consider the coloring of $P_{n+2}$ in Corollary 3.2. Follow the coloring protocol in Proposition 3.3 and without loss of generality let, $c\left(v_{n+3}\right)=c_{3}$. It implies that $c\left(u_{n+3, i}\right)=c_{1}$, $i=1,4,7, \ldots,(m-2), c\left(u_{n+1, i}\right)=c_{2}, i=2,5,8, \ldots,(m-1)$, $c\left(u_{n+1, i}\right)=c_{4}, i=3,6,8, \ldots, m$. Hence, $\theta\left(c_{1}\right)=\theta\left(c_{2}\right)=$ $\theta\left(c_{3}\right)=k(m+1) \frac{2 m}{3}+1, \theta\left(c_{4}\right)=k(m+1)+m$ which is not a perfect Lucky 4-coloring. However, the vertex partition is an optimal near-completion $\chi$-partition. Therefore, the chromatic completion will yield the chromatic completion number.

Also, $\varepsilon\left(P_{n^{\prime}} \circ P_{m}\right)=n(m+1)+7 m+2$. From Lemma 2.1 and Corollary 2.1 it follow that, $\zeta\left(P_{n^{\prime}} \circ C_{m}\right)=3\left[\left(k(m+1)+\frac{2 m}{3}+\right.\right.$ $\left.1)^{2}+\left(k(m+1)+\frac{2 m}{3}+1\right)(k(m+1)+m)\right]-n(m+1)-7 m-$ 2 .

\section{Conclusion}

In Section 3 the family of paths were considered by a partition of order i.e. $n=1,2,3$ and $n=4 k,(4 k+1),(4 k+$ $2),(4 k+3), k=1,2,3, \ldots$ Corona'd to these paths $P_{n}$, only the cycles $C_{m}$ of order $m \in O_{1}$ were considered. It is the author's considered view that the methodology has been well established in this paper. Therefore, deriving the results for the cycles of order $m \in O_{2}$ and $m \in O_{3}$ Corona'd with the path partitions respectively, remain an exercise for the reader.

Conjecture: $\zeta\left(C_{n} \circ P_{m}\right)=\zeta\left(P_{n} \circ P_{m}\right)-1$ and $\zeta\left(C_{n} \circ C_{m}\right)=$ $\zeta\left(P_{n} \circ C_{m}\right)-1$. Prove or disprove the conjecture.

It is deemed worthy research to find results for other known graph operations. 


\section{Acknowledgments}

The author would like to express sincere gratitude to the reviewers for his/her valuable suggestions.

\section{References}

[1] J.A. Bondy and U.S.R. Murty. Graph Theory with Applications. Macmillan Press, London, 2017.

[2] F. Harary. Graph Theory. Addison-Wesley, Reading MA, 1969.

[3] J. Kok. Chromatic completion number. Communicated.

[4] J. Kok. Stability in respect of chromatic completion of graphs. Communicated.

[5] B. West. Introduction to Graph Theory. Prentice-Hall, Upper Saddle River, 1996. 